\title{
Tuning optical properties of MOF-based thin films by changing the ligands of MOFs
}

\author{
Wenchang Yin ${ }^{1}$, Cheng-an Tao ${ }^{1 *}$, Fang Wang ${ }^{1}$, Jian Huang ${ }^{1}$, Tianliang $\mathrm{Qu}^{2}$ and Jianfang Wang ${ }^{{ }^{*}}$
}

\begin{abstract}
The preparation and development of novel optical thin films are of great importance to functional optical and opto-electric components requiring a low refractive index. In this study, a typical metal-organic framework (MOF), MIL$101(\mathrm{Cr})$, is selected as the research model. The corresponding MOF nanoparticles are prepared by a hydrothermal method and the optical thin films are successfully prepared by spincoating. The optical properties of the corresponding MOF thin films are controlled by changing the type of functional groups on the benzene ring of the ligand (terephthalic acid) on MOFs. The functional groups are hydrogen atoms $(\mathrm{H})$, electron donating groups $\left(-\mathrm{NH}_{2},-\mathrm{OH}\right)$ and electron withdrawing groups $\left(-\mathrm{NO}_{2},-\left(\mathrm{NO}_{2}\right)_{2}\right.$ or $\left.\mathrm{F}_{4}\right)$, respectively. It is found that the effective refractive index $\left(n_{\text {eff }}\right)$ of MOF thin films decreases along with the increasing voids among MOF nanoparticles. In addition, the extinction coefficient $(k)$ increases with the addition of electron donating groups, and decreases with the addition of electron withdrawing groups. Among the MOFs used in this study, the $n_{\text {eff }}$ of $\mathrm{NO}_{2}$-MIL-101(Cr) containing electron withdrawing groups is as low as $\sim 1.2$, and value of $k$ is particularly low, which suggests its potential application in antireflective devices. In addition, the intrinsic refractive index $\left(n_{\text {dense }}\right)$ of the dense MOF materials evaluated according to their porosity increases with the number of the functional groups, and the $n_{\text {dense }}$ of the two nitro-substituted MOFs is greater than that of the single nitro-substituted one, and the latter is bigger than that of hydroxyl-substituted one, which is close to that of amino-functionalized one. The diversity of ligands in MOFs makes them a promising new generation of optical materials.
\end{abstract}

Keywords: metal-organic frameworks, thin film, optical property, refractive index, changing of ligand

\section{INTRODUCTION}

Optical thin films are comprised of layered media with uniform thicknesses. They form the basis of developing a variety of optical elements and devices including highreflectivity coatings, antireflective films, polarizing films, long-pass and short-pass filters, and beam splitters [1,2]. The refractive index (RI) is a very significant parameter for optical thin films. For example, antireflective films are usually used to reduce transmission losses. The RI of the film must be equal to $\left(n_{\mathrm{a}} \cdot n_{\mathrm{s}}\right)^{1 / 2}$, where $n_{\mathrm{a}}$ and $n_{\mathrm{s}}$ are the refractive indexes of the air and the substrate, in order to obtain approximately zero reflection at a particular wavelength [3]. Transparent optical glass with a RI of about 1.5 is the most common substrate. In this case, antireflective coatings with a RI of $\sim 1.22$ are required to achieve zero reflection $[3,4]$. Unfortunately, this is nearly impossible to be satisfying because of the lack of optical materials with such a low RI. In fact, the lowest RI that can be achieved in dense materials is only $1.38\left(\mathrm{MgF}_{2}\right)$ [5]. Therefore, the search for materials with a low RI has been one of the most important issues in the field of optical thin films.

Metal-organic frameworks (MOFs) are a kind of new inorganic-organic hybrid materials. These are crystalline materials consisting of metal ions or clusters and organic linker(s) with strong covalent bonds [6,7]. Due to their high porosity, large internal surface area, and high thermal stability (generally $>300^{\circ} \mathrm{C}$ ) [7], MOFs have a variety of potential applications in many fields including gas storage [8], catalysis $[9,10]$, drug delivery [11], and chemical sensing [12-14]. The intrinsic ultra-high porosity of MOFs may lead to a low RI of MOF materials. In addition, over 20,000 types of MOFs exist [15], and their properties can be tuned through the adoption of different metal motifs, organic ligands or crystal morphologies [16]. These characteristics make MOFs a new generation of optical thin film materials.

Consequently, some efforts [17-25] have been made to

${ }^{1}$ College of Science, National University of Defense Technology, Changsha 410073, China

${ }^{2}$ College of Optoelectronic Science and Engineering, National University of Defense Technology, Changsha 410073, China

* Corresponding authors (emails: tca02@mails.thu.edu.cn (Tao C); wangjianfang@nudt.edu.cn (Wang J)) 
explore the application of MOFs in optical thin films. Férey and coworkers [17] first described a simple route for the preparation of flexible porous iron carboxylate MIL-89 optical thin films using the dip-coating method. Then they reported MIL-101(Cr) [18], MIL-100(Cr) [19] and MIL-100(Fe) [19] optical thin films through the same method. Redel et al. [20] presented the optical properties of surface-anchored MOFs, HKUST-1, and determined an optical constant of $n=1.39$ at a wavelength of $750 \mathrm{~nm}$. $\mathrm{Lu}$ et al. [21] constructed ZIF-8-based Fabry-Pérot devices via a direct growth method that functions as selective sensors for chemical vapors and gases. Ranft et al. [22] fabricated tandem MOF-based photonic crystals for enhanced analyte-specific optical detection by spin-coating. Our group $[23,24]$ also fabricated flexible MOF-based photonic films for sensing. These studies focused on the reversible responses of MOFs on an external stimulus. However, the effect of the ligand with different functional groups on the optical properties of the MOF thin film itself has not been explored.

In this study, a commonly used MOF, MIL-101(Cr) (MIL: Materials from Institute Lavoisier) was selected as a model due to its high porosity and large internal surface area. Six ligands with different groups: hydrogen atoms $(\mathrm{H})$, electron donating groups $\left(-\mathrm{NH}_{2},-\mathrm{OH}\right)$ and electron withdrawing groups $\left(-\mathrm{NO}_{2},-\left(\mathrm{NO}_{2}\right)_{2}\right.$ or $\left.-\mathrm{F}_{4}\right)$, were chosen to explore the effect of the ligands on the optical properties of MOF thin films. Five isostructural MOFs with different ligands in the nanoscale were successfully hydrothermally synthesized and characterized in detail. MOF-based optical thin films were fabricated by the spincoating method. The effective RIs $\left(n_{\text {eff }}\right)$ and the extinction coefficients $(k)$ of MOF thin films were analyzed by ellipsometry. The intrinsic RIs of dense MOF materials $\left(n_{\text {dense }}\right)$ were also evaluated. The addition of ligands on the MOF caused the $n_{\text {eff }}$ of the thin film to decrease and the $n_{\text {dense }}$ of the thin film to increase. Specifically, $\mathrm{NO}_{2}$-MIL$101(\mathrm{Cr})$ optical thin film is a promising candidate for various optical and opto-electronic devices due to its low $n_{\text {eff }}(\sim 1.20)$ and low $k$.

\section{EXPERIMENTAL SECTION}

\section{Materials}

$\mathrm{Cr}\left(\mathrm{NO}_{3}\right)_{3} \cdot 9 \mathrm{H}_{2} \mathrm{O}$ (97\%), terephthalic acid $\left(\mathrm{H}_{2} \mathrm{BDC}, 99 \%\right)$ and 2-aminoterephthalic acid $\left(\mathrm{H}_{2} \mathrm{~N}-\mathrm{BDC}, 99 \%\right)$ were purchased from Alfa Aesar Chemical. 2-Hydroxyterephthalic acid (OH-BDC, 99\%), 2-nitroterephthalic acid $\left(\mathrm{O}_{2} \mathrm{~N}-\mathrm{BDC}, 99 \%\right)$ and 2,5-dinitroterephthalic acid $\left(\left(\mathrm{NO}_{2}\right)_{2}-\mathrm{BDC}, 99 \%\right)$ were purchased from Chemsoon
Chemical (Shanghai, China). 2,3,5,6-Tetrafluoroterephthalic acid ( $\left.\mathrm{F}_{4}-\mathrm{BDC}, 99 \%\right)$ was purchased from Adamas Reagent (Shanghai, China). All other chemicals were from a local supply house. All reagents were used as received without further purification. Deionized water was used throughout the work. Silicon wafers $(2 \mathrm{~cm}$ $\times 2 \mathrm{~cm}$ ) were used as substrates and were pre-cleaned with soap and water and subsequently treated with piranha solution $\left(\mathrm{H}_{2} \mathrm{SO}_{4} / \mathrm{H}_{2} \mathrm{O}_{2}\right.$, volumetric ratio 7:3). After thoroughly rinsing with deionized water, the wafers were dried under hot air flow and stored in ethanol.

\section{Fabrication of MOF-based optical thin film}

MIL-101(Cr), $\quad \mathrm{NH}_{2}$-MIL-101(Cr), $\mathrm{NO}_{2}$-MIL-101(Cr), OH-MIL-101(Cr), $\left(\mathrm{NO}_{2}\right)_{2}$-MIL-101(Cr), and $\mathrm{F}_{4}$-MIL-101 $(\mathrm{Cr})$ were synthesized according to the reported method with modification [26,27]. The particles of these MOFs are all in the nanoscale. The nanoscale MOFs suspensions were prepared by dispersing these six kinds of MOF powders in EtOH. The thin films were fabricated by spincoating $200 \mu \mathrm{L}$ of MOF alcoholic suspensions with MOFs content of 3.0-4.0 wt.\% onto the substrate at 3,000 rpm for $60 \mathrm{~s}$. In detail, $4.0 \mathrm{wt}$ \% for MIL-101(Cr), $\mathrm{NH}_{2}$-MIL101(Cr) and $\mathrm{NO}_{2}$-MIL-101(Cr), 3.2\% for OH-MIL-101 (Cr), 3.0\% for $\left(\mathrm{NO}_{2}\right)_{2}$-MIL-101(Cr), and $3.1 \%$ for $\mathrm{F}_{4}$-MIL$101(\mathrm{Cr})$. The films were annealed at $200^{\circ} \mathrm{C}$ for $20 \mathrm{~min}$ after coating.

\section{Characterization}

Transmission electron microscopy (TEM) micrographs were obtained using a JEOL JEM 1230 electron microscope with an accelerating voltage of $200 \mathrm{kV}$. Fourier transform infrared (FT-IR) spectra were obtained using a Spectra Two spectrophotometer (PerkinElmer, USA) from 4,000 to $400 \mathrm{~cm}^{-1}$ with an attenuated total reflection (ATR) accessory. The powder X-ray diffraction (XRD) patterns were collected using a TTR III type X-ray diffractometer (Rigaku, Japan) in $\theta-\theta$ geometry from $5^{\circ}$ to $50^{\circ}(2 \theta)$ with a graphite-monochromated $\mathrm{Cu}-\mathrm{K} \alpha$ radiation source. The $\mathrm{N}_{2}$ adsorption-desorption isotherm of the samples at liquid nitrogen temperature $(78 \mathrm{~K})$ and gas saturation vapor tension range were measured by a BEL Mini sorption instrument. Size distribution was measured using a NICOMP 380 dynamic light scattering (DLS) instrument. A fiber spectrometer (USB2000+, Ocean optics, USA) coupled to optical microscope was used to measure the specular reflectance in the $380-900 \mathrm{~nm}$ range at the normal incidence. Scanning electron microscopy (SEM) images were obtained using a Quanta FEG250 electron microscope with a primary electron energy of 

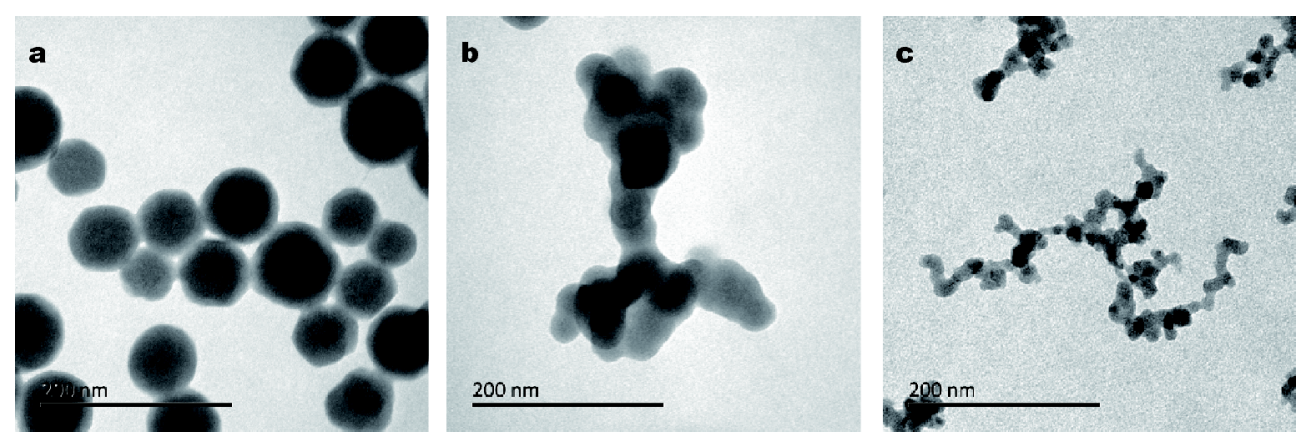

Figure 1 TEM images of MIL-101(Cr) (a), $\mathrm{NH}_{2}-\mathrm{MIL}-101(\mathrm{Cr})$ (b) and $\mathrm{NO}_{2}$-MIL-101(Cr) (c).

$10.0 \mathrm{kV}$. The samples were sputtered with a thin layer of $\mathrm{Au}$ prior to imaging. The surface profiles were characterized using a profilometer (Talysurf PGI 1240, Taylor-Hobson, UK) with a diamond stylus. Ellipsometry measurements were performed with a XLS-100 ellipsometer (Woollam) at $65^{\circ}, 70^{\circ}, 75^{\circ}$, and within a spectral range of $400-800 \mathrm{~nm}$ at room temperature.

\section{RESULTS AND DISCUSSION}

\section{Preparation of nanoscale MOFs}

The nanoscale MIL-101(Cr), $\mathrm{NH}_{2}$-MIL-101(Cr) and $\mathrm{NO}_{2}-$ MIL-101(Cr) were obtained using the hydrothermal method [26,27]. From the TEM images (Fig. 1), the sizes of the three MOFs are about 66,40 , and $14 \mathrm{~nm}$, respectively (Fig. S1-S3). MIL-101(Cr) nanoparticles are nearly spherical, while $\mathrm{NH}_{2}$-MIL-101(Cr) and $\mathrm{NO}_{2}$-MIL-101(Cr) have an irregular shape. Both $\mathrm{NH}_{2}-\mathrm{MIL}-101(\mathrm{Cr})$ and $\mathrm{NO}_{2}$-MIL-101(Cr) exhibit the phenomenon of aggregation due to too small sizes. The nanoscale MOFs were characterized by FT-IR, XRD, $\mathrm{N}_{2}$ adsorption-desorption isotherms, and DLS (Fig. 2). Compared with FT-IR spectra of $\mathrm{H}_{2} \mathrm{BDC}, \mathrm{NH}_{2}-\mathrm{BDC}$ and $\mathrm{NO}_{2}$ - $\mathrm{BDC}$ (Fig. 2a-c), all the bands of protonated carboxylic groups at around $1,710 \mathrm{~cm}^{-1}$ disappear in these three MOFs, indicating that all of the carboxyl groups were coordinated with $\mathrm{Cr}^{3+}$ [28]. The IR spectra of $\mathrm{NH}_{2}$-MIL-101(Cr) show the vibrational bands of the amino-groups at around 3,337 and $3,467 \mathrm{~cm}^{-1}[29,30]$. The bands at around 1,352 and 1,545 $\mathrm{cm}^{-1}$ confirm the existence of nitro-groups in $\mathrm{NO}_{2}$-MIL101(Cr) $[29,30]$. The observed powder XRD patterns of these three MOFs (Fig. 2d) are consistent with the crystal structure presented in the literature [31], which confirms the formation of pure MOFs. The $\mathrm{N}_{2}$ adsorption-desorption isotherms of MIL-101(Cr), $\mathrm{NH}_{2}$-MIL-101(Cr) and $\mathrm{NO}_{2}$-MIL-101(Cr) are shown in Fig. 2e. The isotherms of $\mathrm{NH}_{2}$-MIL-101(Cr) and $\mathrm{NO}_{2}$-MIL-101(Cr) show a hysteresis loop respectively meaning that there is a large number of mesopores due to the packing of the nanoscale particles. The BET surface areas of MIL-101(Cr), $\mathrm{NH}_{2}$ MIL-101(Cr) and $\mathrm{NO}_{2}$-MIL-101(Cr) samples were calculated to be $2,760,2,322$ and $521.6 \mathrm{~m}^{2} \mathrm{~g}^{-1}$, respectively. Based on the desorption isotherms, the pore size of both MOFs was evaluated by the MP method and BJH method, respectively (Table S1). The micropore sizes of both MIL$101(\mathrm{Cr})$ and $\mathrm{NH}_{2}$-MIL-101(Cr) evaluated by the MP method are $2 \mathrm{~nm}$, due to the $-\mathrm{NH}_{2}$ group's small volume, which has no effect on the internal pore sizes. However, the pore size of $\mathrm{NO}_{2}-\mathrm{MIL}-101(\mathrm{Cr})$ is only $1.2 \mathrm{~nm}$, suggesting the strong stereo-hindrance effect of the $-\mathrm{NO}_{2}$ group on the pore size. Consequently, the micropore volume and the size of the substituent group of the MOFs decrease. The mesopore size distribution of the MOFs evaluated with the BJH method is shown in Table S1. All the three MOFs have $3.3 \mathrm{~nm}$ pores, which is typical of the larger internal cages of MIL-101. There are also packing mesopores in $\mathrm{NH}_{2}-\mathrm{MIL}-101(\mathrm{Cr})$ of about $44.2 \mathrm{~nm}$ and $\mathrm{NO}_{2}$-MIL-101(Cr) aggregates of about 15.9 and $32.5 \mathrm{~nm}$. The mesopore volume of MIL-101(Cr) is very small, but those of $\mathrm{NH}_{2}$-MIL-101(Cr) and $\mathrm{NO}_{2}$-MIL-101(Cr) increase significantly. According to DLS measurements (Fig. 2f), the particle sizes of MIL-101(Cr), $\mathrm{NH}_{2}$-MIL-101 $(\mathrm{Cr})$ and $\mathrm{NO}_{2}-\mathrm{MIL}-101(\mathrm{Cr})$ are about 43,55 , and $37 \mathrm{~nm}$, respectively, which are larger than the results of the TEM measurements due to solvation in $\mathrm{EtOH}$. These results indicate that nanoscale MIL-101(Cr), $\mathrm{NH}_{2}$-MIL-101(Cr) and $\mathrm{NO}_{2}$-MIL-101(Cr) have been successfully prepared, and these three MOFs have a large number of pores and large specific surface areas. These properties facilitate the formation of MOFs films with low RIs.

\section{Fabrication of MOF-based optical thin films}

The MOF-based optical thin films were fabricated by the spin-coating method. Previously, we showed that the thickness of the thin film could be adjusted by altering the concentration of the MOF suspension, the spin-coating 

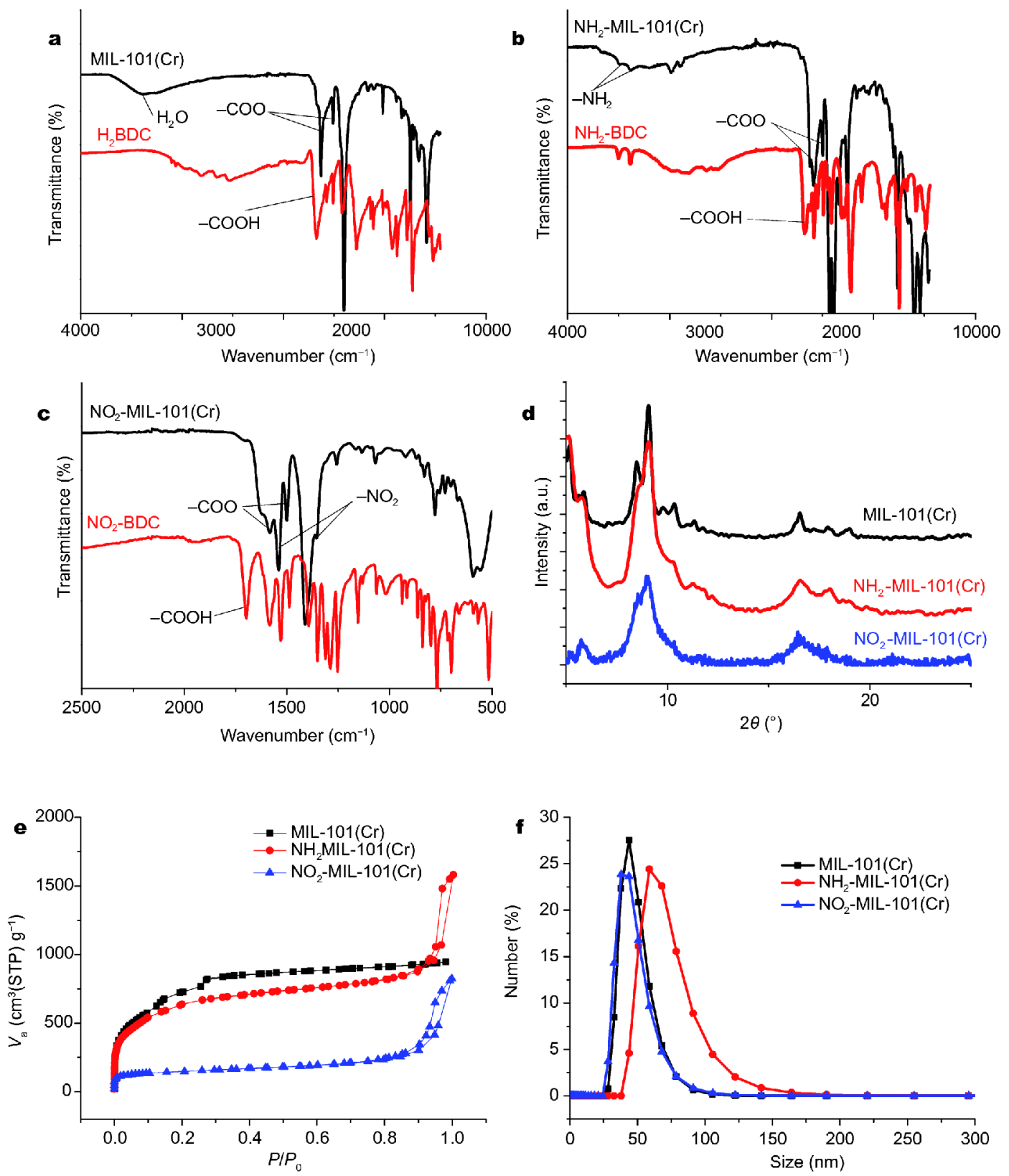

Figure 2 FT-IR spectra of MIL-101(Cr) (a), $\mathrm{NH}_{2}$-MIL-101(Cr) (b), and $\mathrm{NO}_{2}$-MIL-101(Cr) (c); XRD patterns (d); $\mathrm{N}_{2}$ adsorption-desorption isotherms (e) and DLS (f).

rotation speed and spin-coating times [23,24], while the optical parameters kept constant. To get the thin film with moderate thickness $(<1000 \mathrm{~nm})$, in this study, these three factors were controlled to be 3.0-4.0 wt.\%, 3000 rpm and only once. Fig. 3 shows photographs and reflectance spectra of fabricated MOF optical films, which display orchid MIL-101(Cr), violet $\mathrm{NH}_{2}-\mathrm{MIL}-101(\mathrm{Cr})$ and light blue $\mathrm{NO}_{2}$-MIL-101(Cr). These vivid colors indicate the high-quality of MOF-based optical thin films.
In addition, the reflectance spectra of the films are consistent with the color of the films. The reflection peaks of MIL-101(Cr), $\mathrm{NH}_{2}-\mathrm{MIL}-101(\mathrm{Cr})$ and $\mathrm{NO}_{2}$-MIL-101(Cr) are 440, 477 and $490 \mathrm{~nm}$, respectively. Compared with MIL-101(Cr), the reflection peaks of $\mathrm{NH}_{2}$-MIL-101(Cr) and $\mathrm{NO}_{2}$-MIL-101(Cr) red shift, and the reflectance at $600 \mathrm{~nm}$ reduces from $90 \%$ to $60 \%$ and $68 \%$, respectively.

These differences in color and reflectivity may be attributed to the change in the RI and the thickness of the 

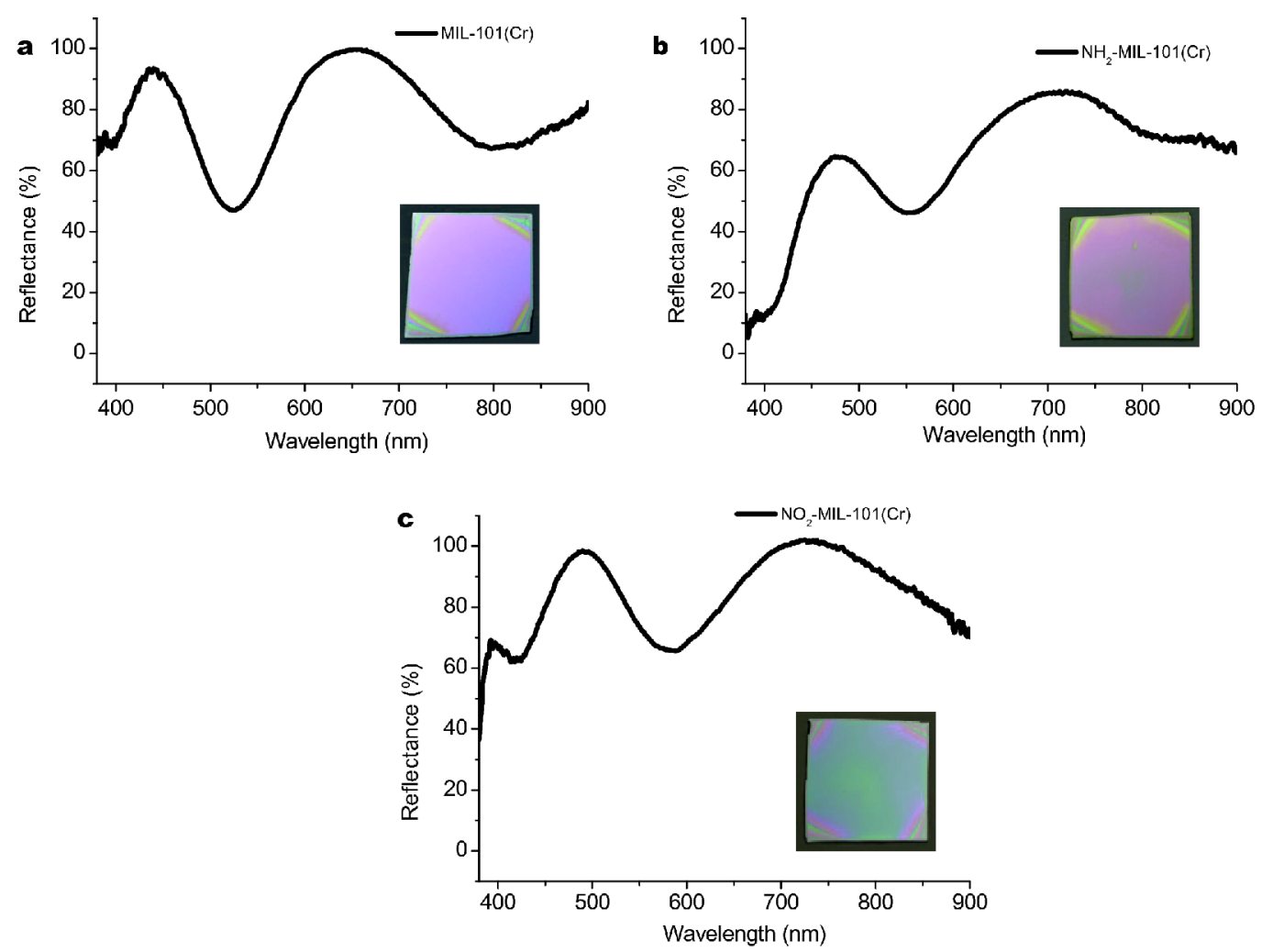

Figure 3 Photographs and reflection spectra of MIL-101(Cr) (a), $\mathrm{NH}_{2}-\mathrm{MIL}-101(\mathrm{Cr})$ (b) and $\mathrm{NO}_{2}$-MIL-101(Cr) (c) MOF optical thin films.

films. From the side-view SEM images (Fig. 4a-c), the thickness of $\mathrm{NO}_{2}$-MIL-101(Cr) is $634 \mathrm{~nm}$, which is a little larger than those of MIL-101(Cr) and $\mathrm{NH}_{2}$-MIL-101(Cr). MIL-101(Cr) and $\mathrm{NH}_{2}$-MIL-101(Cr) have similar thicknesses, which are 509 and $521 \mathrm{~nm}$, respectively. Thus, the significant changes in color of MIL-101(Cr) and $\mathrm{NO}_{2}$ MIL-101(Cr) are due to the RI of the films rather than the small changes in thickness. Also, the precise thicknesses of MIL-101(Cr), $\mathrm{NH}_{2}$-MIL-101(Cr) and $\mathrm{NO}_{2}$-MIL-101 (Cr) were further evaluated by ellipsometry to be 515,530 and $630 \mathrm{~nm}$, respectively, which are very close to those obtained from the SEM measurements. There are some cracks on the surface of the films, which are caused by an induced tensile stress during the thermal annealing process, especially in the film of $\mathrm{NH}_{2}-\mathrm{MIL}-101(\mathrm{Cr})$ (Fig. 4e). The cracks are all on nanoscale which has no effect on the macroscale surface smoothness. However, these nanoscale gaps and cavities can efficiently increase the porosity of the films, which results in a low RI according to the effective medium theory $[32,33]$.

The surface roughness were determined using a profilometer with a diamond stylus. The roughness profiles of MOF-based thin films are shown in Fig. S4. The ar- ithmetic average roughness, $R_{\mathrm{a}}$ is the arithmetic average value of filtered roughness profile determined from deviations about the center line within the evaluation length and calculated from the formula,

$$
R_{\mathrm{a}}=\frac{1}{n} \sum_{i=1}^{n}\left|y_{i}\right|,
$$

and the root mean squared roughness, $R_{\mathrm{q}}$ is root mean squared value of filtered roughness profile based on the formula

$$
R_{\mathrm{q}}=\sqrt{\frac{1}{n} \sum_{i=1}^{n} y_{i}^{2}}
$$

where $n$ is the ordered, equally spaced points along the trace, $y_{\mathrm{i}}$ is the vertical distance from the mean line to the $i^{\text {th }}$ data point. The calculated $R_{\mathrm{a}}$ and $R_{\mathrm{q}}$ are listed in Table S2. The $R_{\mathrm{a}}$ of MIL-101(Cr), $\mathrm{NH}_{2}-\mathrm{MIL}-101(\mathrm{Cr})$, and $\mathrm{NO}_{2}$-MIL-101(Cr) thin films are only 3.4, 3.0, and 4.2 $\mathrm{nm}$, respectively. The $R_{\mathrm{q}}$ of them are $4.4,3.8$, and $5.5 \mathrm{~nm}$, which are smaller than those of MOF-based thin films in previous reports $[18,34,35]$. For instance, Demessence et al. [18] reported that the root-mean-square roughness of MIL-101(Cr) optical thin films was calculated to be around $12 \mathrm{~nm}$ (nearly half of the MOF NP diameter). The 

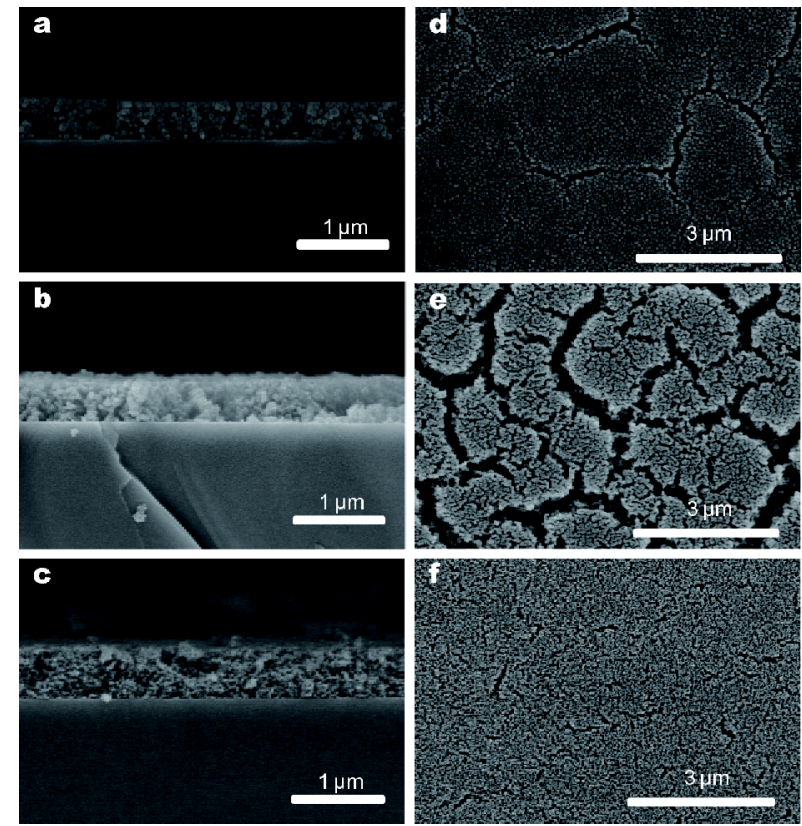

Figure 4 SEM images (a-c: top-view, d-f: side-view) of MIL-101(Cr) (a, d), $\mathrm{NH}_{2}$-MIL-101(Cr) (b, e), and $\mathrm{NO}_{2}$-MIL-101(Cr) (c, f) MOF optical thin films.

root-mean-square surface roughness of gel-layer synthesized Fe-MIL-88B_NH $\mathrm{NH}_{2}$ thin film was 5-9 nm (from atomic force microscope images) [34]. These results suggest that the fabricated MOF optical thin films are of high quality.

\section{Optical analysis of MOF-based optical thin films}

Spectroscopic ellipsometry was used to investigate the effect of the MOFs' ligands on the optical constants of the MOF-based thin films. The ellipsometric parameters ( $P s i$, Delta) were obtained in the wavelength range of 400-800 $\mathrm{nm}$ at three incident angles $65^{\circ}, 70^{\circ}$ and $75^{\circ}$, respectively.

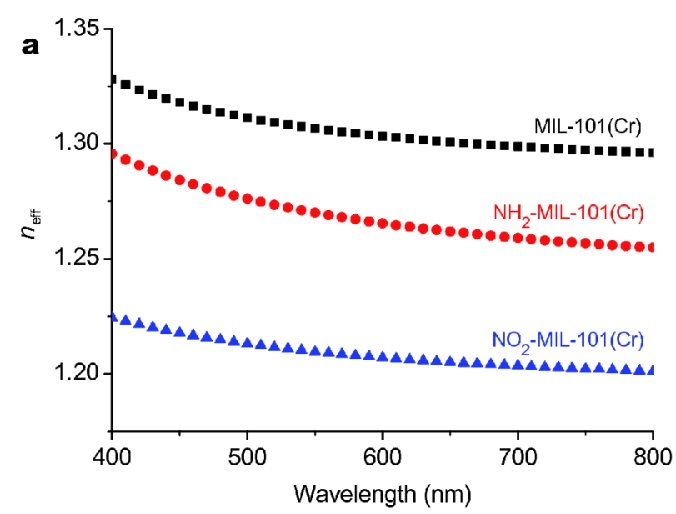

Figure 5 The $n_{\text {eff }}$ (a) and $k$ (b) of MOFs optical thin films.
In data reduction, a proper theoretical dispersion model should be chosen to obtain the optical constants of the thin films. To simplify the data fitting procedure, a Cauchy model $[18,20]$ was chosen to fit the experimental data. The measured and fitted ellipsometric parameters of MIL-101(Cr), $\mathrm{NH}_{2}-\mathrm{MIL}-101(\mathrm{Cr})$ and $\mathrm{NO}_{2}$-MIL-101(Cr) are presented in Figs S5-S7. The fitting of MOF thin films presents sufficient agreement with the experimental data over the entire measured spectral range, with a factor of root-mean-square error $\approx 15.99,20.06$ and 17.60 , demonstrating the accuracy and reliability of the data fitting procedure.

Fig. 5 shows the optical constants reduced from the fitting of ellipsometric data using the Cauchy model. Both the $n_{\text {eff }}$ and $k$ can be properly tuned by adding functional groups on the ligands. The average $n_{\text {eff }}$ of MIL-101(Cr) and $\mathrm{NH}_{2}$-MIL-101(Cr) are 1.306 and 1.268 (the average RI from 400 to $800 \mathrm{~nm}$ ) respectively. $\mathrm{NO}_{2}$-MIL-101(Cr) has the lowest $n_{\text {eff }}$ with a value of 1.208 , which is significantly lower than 1.22 . The low $n_{\text {eff }}$ in the $\mathrm{NO}_{2}$-MIL$101(\mathrm{Cr})$ optical thin film shows its potential applications in antireflective films and other optical devices $[3,4]$. With the addition of the ligand functional groups, the thin film's $n_{\text {eff }}$ in the spectral range of $400-800 \mathrm{~nm}$ are reduced, which is largely due to the increasing voids among the MOF nanoparticles. The value of $k$ increases with the addition of electron-donating groups $\left(-\mathrm{NH}_{2}\right)$ and decreases with the addition of electron-withdrawing group $\left(-\mathrm{NO}_{2}\right)$. The very low $k$ value in the $\mathrm{NO}_{2}-\mathrm{MIL}-101$ (Cr) optical thin film suggests low absorption in the visible spectrum, which can be used in antireflective film applications.

To understand the effect of the ligand on the $n_{\text {dense }}$ of MOF materials, the effective medium theory [36] was used to further evaluate the value of $n_{\text {dense. }}$ The pore size of these MOFs films is all in the nanoscale (less than 100

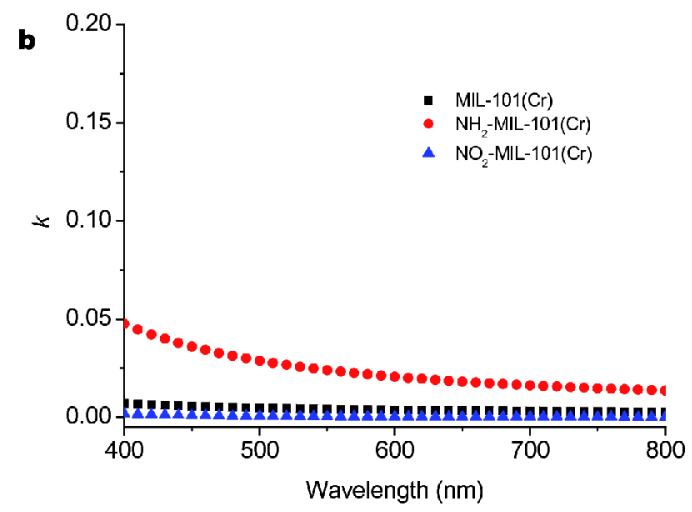



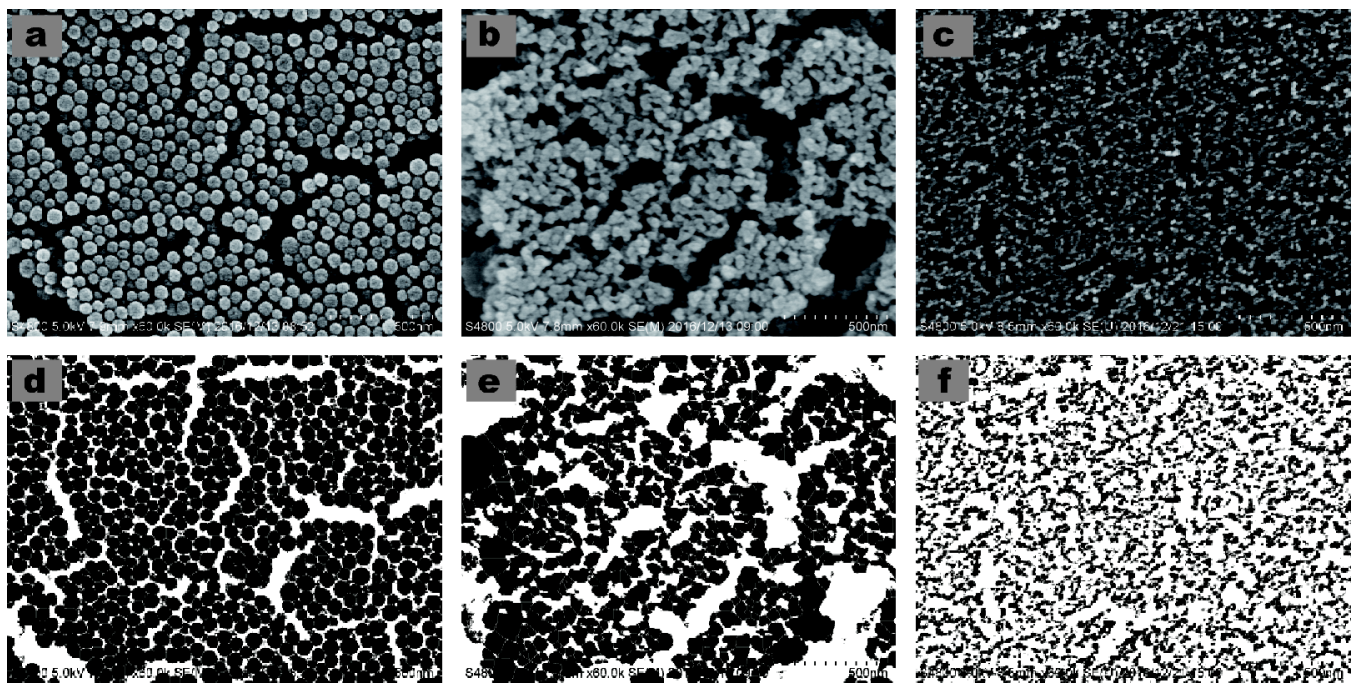

Figure 6 The original SEM images and binary images of MIL-101(Cr) (a, d), $\mathrm{NH}_{2}$-MIL-101(Cr) (b, e), and NO $\mathrm{N}_{2}-\mathrm{MIL}-101$ (Cr) (c, f) MOF optical thin films.

$\mathrm{nm})$ and are much smaller than the wavelength of the light, which satisfies the theory well. Also, the $n_{\text {eff }}$ of the films must follow Equation (3), where $n_{\text {eff }}$ and $n_{\text {dense }}$ are the RIs of porous materials and dense materials, respectively, and $f$ represents the porosity [36]. If the $n_{\text {eff }}$ value and the $f$ of the films have been obtained, the $n_{\text {dense, }}$ which represents the RIs of dense MOFs can be calculated according to Equation (3).

$$
n_{\text {eff }}^{2}=n_{\text {air }}^{2} f+n_{\text {dense }}^{2}(1-f),
$$

The $f$ of the MOFs packing structure was evaluated based on their SEM images by image processing and statistical analysis with Image-J software. As shown in Fig. 6, it is obvious that the nanoparticles on the silicon are in different gray levels with the gaps and cavities. Thus, it is possible to translate the SEM pictures (Fig. 6ac) into black and white binary images (Fig. 6d-f). The integration can be used to evaluate the porosities caused by stacked nanoparticles. The stacked porosities of MIL101(Cr), $\mathrm{NH}_{2}-\mathrm{MIL}-101(\mathrm{Cr})$ and $\mathrm{NO}_{2}$-MIL-101(Cr) are $28.4 \%, 42.9 \%$ and $64.9 \%$, respectively. The morphology and size of these three MOF particles were determined to be accumulated in different stack modes and resulted in different porosities. The spherical close-packed particles of MIL-101(Cr) result in only a few tiny gaps on the surface. In contrast, $\mathrm{NH}_{2}$-MIL-101(Cr) shows larger gaps and cavities due to the closer accumulation of the particles. This is because of the strong hydrophilicity of the amino group, which promotes the aggregation degrees during the thermal annealing process. The $\mathrm{NO}_{2}$-MIL-101 (Cr) film shows higher porosity due to its complex, net-

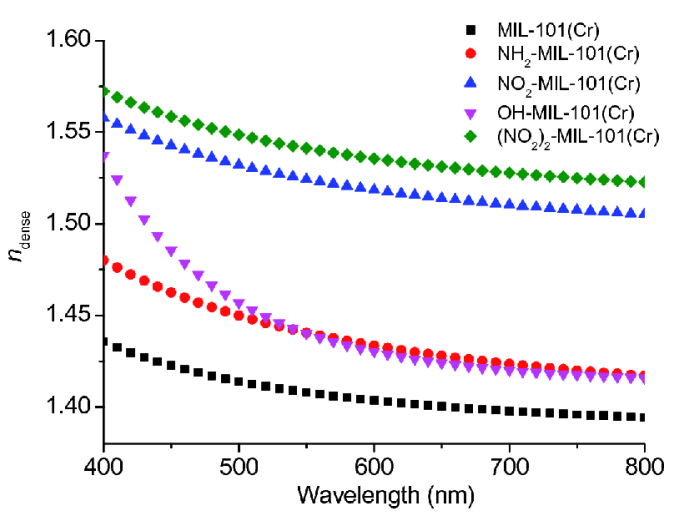

Figure 7 The $n_{\text {dense }}$ of dense MOF materials.

worked structure comprised of connected nanoparticles.

The value of $n_{\text {eff }}$ is shown in Fig. 5. The value of $n_{\text {air }}$ is approximately 1 , and the $n_{\text {dense }}$ can be calculated using Equation (3). The calculated results of $n_{\text {dense }}$ are shown in Fig. 7. The $n_{\text {dense }}$ of MIL-101-(Cr), $\mathrm{NH}_{2}$-MIL-101(Cr) and $\mathrm{NO}_{2}$-MIL-101(Cr) are 1.407, 1.438 and 1.522 (the average RI from 400 to $800 \mathrm{~nm}$ ), respectively. These values indicate that the inherent refractive index of the MOF materials increases with the addition of ligand functional groups, which is attributed to the increase in the atomic density of the MOFs. This confirms that the $n_{\text {eff }}$ of these MOF-based optical thin films are primarily affected by the inter-voids of stacked nanoparticles.

The stability of MOF thin film is critical for practical application. MIL-101(Cr) thin films were taken as an example to evaluate their stability by immersing them in organic solvents or water with different $\mathrm{pH}$ values. As 
shown in Fig. S8, the fabricated thin films can retain their color after immersing in varied organic solvents for 30 min, such as petroleum ether, methylbenzene, trichloromethane, ethanol or dimethyl formamide. The corresponding reflection spectra do not shift (Fig. S9), demonstrating the stability of MOF films. The MIL-101 (Cr) thin film is also of great stability in acidic solution from $\mathrm{pH} 1$ to 7 , since its color and the reflection spectra almost keep constant. The color of them would change when it was immersing in basic solution due to the MIL101 can be digested in basic medium (Figs S10 and S11). The as-fabricated thin films have good stability whatever in organic solvents or acidic solution, and fabrication of optical thin films with more stable MOFs (such as $\mathrm{UiO}$ series) instead of MIL-101 will further improve their stability in basic medium.

The same idea was applied to three other ligands $(\mathrm{OH}-$ BDC, $\left(\mathrm{NO}_{2}\right)_{2}-\mathrm{BDC}$ and $\left.\mathrm{F}_{4}-\mathrm{BDC}\right)$ to verify the trend of inherent refractive index of MIL-101 MOFs changing with the ligands. Photographs of MOF-based thin films are shown in Fig. S12, and the corresponding specular reflections are shown in Fig. S13. These vivid colors and specular reflection spectra suggest the formation of MOFbased optical thin films with high quality. Also the reflectance spectra of the films are consistent with their color. SEM images of MOF-based thin films were shown in Fig. S14. Although there are some cracks on the surface under high magnification, the films are uniform, smooth and homogeneous from macroscopical view. The surface roughness were calculated according to the surface profile tested by a profilometer. The roughness profiles of MOFbased thin films are shown in Fig. S4. The $R_{\mathrm{a}}$ of OH-MIL101(Cr), $\left(\mathrm{NO}_{2}\right)_{2}$-MIL-101(Cr), $\mathrm{F}_{4}$-MIL-101(Cr) thin films are only 5.3, 5.5 and $5.2 \mathrm{~nm}$, respectively. The $R_{\mathrm{q}}$ of them are $11.0,7.9$ and $8.7 \mathrm{~nm}$, which further confirmed the high quality of these thin films. The $n_{\text {eff }}$ and void percentage of new-synthesized MOF thin films were obtained according to above-mentioned method (Figs S15S19). The calculated $n_{\text {dense }}$ of these MOF-based thin films are shown in Fig. 7. The MOFs synthesized with $\mathrm{F}_{4}$-BDC are not in the form of MIL-101 (Figs S20 and S21), so the $n_{\text {dense }}$ of them is not shown here. The $n_{\text {dense }}$ of MIL-101 (Cr), OH-MIL-101(Cr), $\mathrm{NH}_{2}$-MIL-101(Cr), $\mathrm{NO}_{2}$-MIL$101(\mathrm{Cr})$, and $\left(\mathrm{NO}_{2}\right)_{2}$-MIL-101(Cr) are 1.404, 1.430, 1.434, 1.519 and 1.536 at the wavelength of $600 \mathrm{~nm}$ (the center wavelength in the range from 400 to $800 \mathrm{~nm}$ ). The $n_{\text {dense }}$ of OH-MIL-101(Cr) and $\mathrm{NH}_{2}-\mathrm{MIL}-101(\mathrm{Cr})$ are comparable in the spectrum of 500 to $800 \mathrm{~nm}$. The $n_{\text {dense }}$ of nitrofunctionalized MIL-101(Cr) is much bigger than that of amino-substituted one. And the $n_{\text {dense }}$ of two nitro-sub- stituted one is larger than that of single functionalized one. These results confirm that the inherent refractive index of the MOF material increases along with the addition of functional groups of ligands, which are attributed to the increase of atomic density of MOFs.

\section{CONCLUSIONS}

Five isostructural nanoscale MOFs with different ligands $\left(\mathrm{H}, \mathrm{OH}-, \mathrm{NH}_{2^{-}}, \mathrm{NO}_{2^{-}}\right.$and two $\left.\mathrm{NO}_{2}{ }^{-}\right)$have been prepared, and the corresponding MOF-based optical thin films have been successfully prepared via spin-coating. The MOF optical thin films are uniform in color and have homogeneous thickness. The $n_{\text {eff }}$ of the thin films decreases with the addition of ligand functional groups, which is largely due to the increasing voids among MOF nanoparticles. The value of $k$ increases with the addition of amino groups $\left(-\mathrm{NH}_{2}\right)$ and decreases with the addition of nitro groups $\left(-\mathrm{NO}_{2}\right)$. The very low values of $n_{\text {eff }}$ and $k$ in the $\mathrm{NO}_{2}$-MIL-101(Cr) optical thin film promote the application of MOF films in antireflective devices. The inherent refractive index of the MOF material increases with the addition of ligand functional groups, which is attributed to the increase in atomic density. In view of the countless types of MOFs, these can become a new generation of optical materials by choosing the appropriate ligands.

Received 31 July 2017; accepted 18 October 2017; published online 24 November 2017

1 Epstein LI. The design of optical filters. J Opt Soc Am, 1952, 42: 806-808

2 Wen X, Xiong Q. A large scale perfect absorber and optical switch based on phase change material $\left(\mathrm{Ge}_{2} \mathrm{Sb}_{2} \mathrm{Te}_{5}\right)$ thin film. Sci China Mater, 2016, 59: 165-172

3 Schulze M, Lehr D, Helgert M, et al. Transmission enhanced optical lenses with self-organized antireflective subwavelength structures for the UV range. Opt Lett, 2011, 36: 3924

4 Wongcharee K, Brungs M, Chaplin R, et al. Sol-gel processing by aging and pore creator addition for porous silica antireflective coatings. J Sol-Gel Sci Tech, 2002, 25: 215-221

5 Bernsmeier D, Polte J, Ortel E, et al. Antireflective coatings with adjustable refractive index and porosity synthesized by micelletemplated deposition of $\mathrm{MgF}_{2}$ sol particles. ACS Appl Mater Interfaces, 2014, 6: 19559-19565

6 Sindoro M, Yanai N, Jee AY, et al. Colloidal-sized metal-organic frameworks: synthesis and applications. Acc Chem Res, 2014, 47: 459-469

7 Park KS, Ni Z, Côté AP, et al. Exceptional chemical and thermal stability of zeolitic imidazolate frameworks. Proc Natl Acad Sci USA, 2006, 103: 10186-10191

8 Murray LJ, Dincă M, Long JR. Hydrogen storage in metal-organic frameworks. Chem Soc Rev, 2009, 38: 1294-1314

9 Lee JY, Farha OK, Roberts J, et al. Metal-organic framework materials as catalysts. Chem Soc Rev, 2009, 38: 1450-1459 
10 Wu S, Zhu Y, Huo Y, et al. Bimetallic organic frameworks derived $\mathrm{CuNi} /$ carbon nanocomposites as efficient electrocatalysts for oxygen reduction reaction. Sci China Mater, 2017, 60: 654-663

11 Horcajada P, Chalati T, Serre C, et al. Porous metal-organic-framework nanoscale carriers as a potential platform for drug delivery and imaging. Nat Mater, 2010, 9: 172-178

12 Serre C, Mellot-Draznieks C, Surblé S, et al. Role of solvent-host interactions that lead to very large swelling of hybrid frameworks. Science, 2007, 315: 1828-1831

13 Kreno LE, Leong K, Farha OK, et al. Metal-organic framework materials as chemical sensors. Chem Rev, 2012, 112: 1105-1125

$14 \mathrm{Wu}$ Y, Li F, Zhu W, et al. Metal-organic frameworks with a threedimensional ordered macroporous structure: dynamic photonic materials. Angew Chem Int Ed, 2011, 50: 12518-12522

15 Peplow M. Materials science: the hole story. Nature, 2015, 520: $148-150$

$16 \mathrm{Xu} \mathrm{X,} \mathrm{Lu} \mathrm{Y,} \mathrm{Yang} \mathrm{Y,} \mathrm{et} \mathrm{al.} \mathrm{Tuning} \mathrm{the} \mathrm{growth} \mathrm{of} \mathrm{metal-organic}$ framework nanocrystals by using polyoxometalates as coordination modulators. Sci China Mater, 2015, 58: 370-377

17 Patricia H, Christian S, David G, et al. Colloidal route for preparing optical thin films of nanoporous metal-organic frameworks. Adv Mater, 2009, 21: 1931-1935

18 Demessence A, Horcajada P, Serre C, et al. Elaboration and properties of hierarchically structured optical thin films of MIL101(Cr). Chem Commun, 2009, 309: 7149-7151

19 Márquez AG, Demessence A, Platero-Prats AE, et al. Green microwave synthesis of MIL-100(Al, Cr, Fe) nanoparticles for thinfilm elaboration. Eur J Inorg Chem, 2012: 5165-5174

20 Redel E, Wang Z, Walheim S, et al. On the dielectric and optical properties of surface-anchored metal-organic frameworks: a study on epitaxially grown thin films. Appl Phys Lett, 2013, 103: 091903

21 Lu G, Hupp JT. Metal-organic frameworks as sensors: a ZIF-8 based Fabry-Perot device as a selective sensor for chemical vapors and gases. J Am Chem Soc, 2010, 132: 7832-7833

22 Ranft A, Niekiel F, Pavlichenko I, et al. Tandem MOF-based photonic crystals for enhanced analyte-specific optical detection. Chem Mater, 2015, 27: 1961-1970

$23 \mathrm{Hu} \mathrm{Z}$, Tao C, Liu H, et al. Fabrication of an $\mathrm{NH}_{2}-\mathrm{MIL}-88 \mathrm{~B}$ photonic film for naked-eye sensing of organic vapors. J Mater Chem A, 2014, 2: 14222-14227

$24 \mathrm{Hu} \mathrm{Z}$, Tao C, Wang F, et al. Flexible metal-organic frameworkbased one-dimensional photonic crystals. J Mater Chem C, 2015, 3: 211-216

25 Yin W, Tao CA, Zou X, et al. The tuning of optical properties of nanoscale MOFs-based thin film through post-modification. Nanomaterials, 2017, 7: 242

26 Jiang D, Burrows AD, Edler KJ. Size-controlled synthesis of MIL101(Cr) nanoparticles with enhanced selectivity for $\mathrm{CO}_{2}$ over $\mathrm{N}_{2}$. CrystEngComm, 2011, 13: 6916-6919
27 Jiang D, Keenan LL, Burrows AD, et al. Synthesis and post-synthetic modification of MIL-101(Cr)- $\mathrm{NH}_{2}$ via a tandem diazotisation process. Chem Commun, 2012, 48: 12053

28 Férey G, Mellot-Draznieks C, Serre C, et al. A chromium terephthalate-based solid with unusually large pore volumes and surface area. Science, 2005, 309: 2040-2042

29 Modrow A, Zargarani D, Herges R, et al. Introducing a photoswitchable azo-functionality inside Cr-MIL-101- $\mathrm{NH}_{2}$ by covalent post-synthetic modification. Dalton Trans, 2012, 41: 8690-8696

30 Bernt S, Guillerm V, Serre C, et al. Direct covalent post-synthetic chemical modification of Cr-MIL-101 using nitrating acid. Chem Commun, 2011, 47: 2838-2840

31 Jiang D, Burrows AD, Xiong Y, et al. Facile synthesis of crack-free metal-organic framework films on alumina by a dip-coating route in the presence of polyethylenimine. J Mater Chem A, 2013, 1: 5497-5500

32 Garnett JCM. Colours in metal glasses, in metallic films, and in metallic solutions. II. Philos Trans R Soc A-Math Phys Eng Sci, 1906, 205: 237-288

33 Bruggeman DAG. Dielectric constant and conductivity of mixtures of isotropic materials. Ann Phys, 1935, 24: 636-679

34 Schoedel A, Scherb C, Bein T. Oriented nanoscale films of metalorganic frameworks by room-temperature gel-layer synthesis. Angew Chem Int Ed, 2010, 49: 7225-7228

35 Stavila V, Schneider C, Mowry C, et al. Thin film growth of nbo MOFs and their integration with electroacoustic devices. Adv Funct Mater, 2016, 26: 1699-1707

36 Li X, Yu X, Han Y. Polymer thin films for antireflection coatings. J Mater Chem C, 2013, 1: 2266-2285

Acknowledgements This work was financially supported by the National Natural Science Foundation of China (21203247 and 21573285) and research project of National University of Defense Technology (ZK16-03-51). We thank LetPub (www.letpub.com) for its linguistic assistance during the preparation of this manuscript.

Author contributions Yin W performed the experiments, analyzed the data, and wrote the paper; Tao C conceived and designed the experiments, and revised the paper; Wang $\mathrm{F}$ and Huang $\mathrm{J}$ analyzed the data; Qu T contributed to Ellipsometry measurements; Wang J conceived and designed the experiments, and analyzed the data. All authors contributed to the general discussion.

Conflict of interest The authors declare that they have no conflict of interest.

Supplementary information Measured and fitted (line) ellipsometry data and pore distribution data are available in the online version of the paper. 


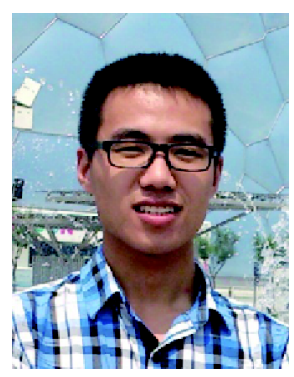

Wenchang Yin received his bachelor degree from Jilin University (JLU) in 2015. He is currently pursuing his master degree under the supervision of Assoc. Prof. Cheng-an Tao and Prof. Jianfang Wang at the National University of Defense Technology (NUDT). His research interests are metal-organic framework materials and their optical properties and applications.

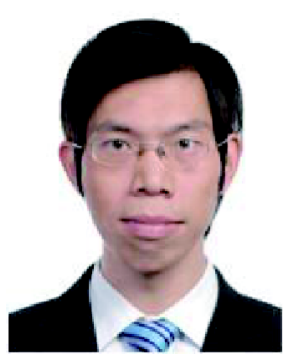

Cheng-an Tao is an associate professor at the College of Science, NUDT. He graduated from Tsinghua University (THU) in 2006 and received his PhD degree at THU in 2011. Then he works at the NUDT until now. His research interest focuses on the porous materials and their application in optical and electrical systems and devices.

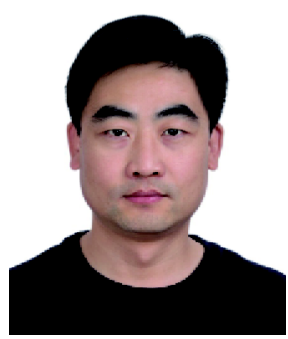

Jianfang Wang is a professor at the College of Science, NUDT. He graduated from the NUDT in 1997 and received his $\mathrm{PhD}$ degree at the NUDT in 2003. Then he works at the NUDT until now. His research interest focuses on nanochemsitry and nanomaterials.

\section{配体改变法调控MOFs薄膜的光学性质}

殷文昌，陶呈安 ${ }^{1^{*}}$, 王芳 ${ }^{1}, \mathbf{M}^{\text {坚 }}{ }^{1}$ 曲天良 ${ }^{2}$, 王建方 ${ }^{1^{*}}$

摘要 制备和开发新型光学薄膜材料对于功能性的光学和光电器件具有重要意义, 特别是具有低折射率的光学薄膜材料. 本论文选用一 类典型的MOFs材料, MIL-101(Cr), 为研究对象, 通过水热法制备得到相应的MOFs纳米颗粒, 并通过旋涂法成功制备光学薄膜. 通过改变 配体(对苯二甲酸)苯环上功能团的种类: 氢原子 $(\mathrm{H})$ 、给电子基团 $\left(-\mathrm{NH}_{2},-\mathrm{OH}\right)$ 和吸电子基团 $\left(-\mathrm{NO}_{2}\right.$, 双 $\left.-\mathrm{NO}_{2}\right)$, 来调控对应的 $\mathrm{MOF}$ 光学薄 膜的光学性质. 研究发现, MOFs 薄膜的有效折射率 $n_{\mathrm{eff}}$ 随着MOFs纳米颗粒之间的孔隙的增加而减小, MOFs薄膜的消光系数 $k$ 随着给电子 基团的增加而增大, 而随着吸电子基团的增加而减小. 其中, 含有吸电子基团的MOF即 $\mathrm{NO}_{2}-\mathrm{MIL}-101(\mathrm{Cr})$ 的折射率最小, 低至1.2左右, 而且

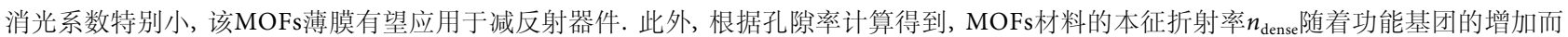
增大，而且双硝基取代的MOFs的折射率大于硝基取代的，大于差基取代的折射率，而差基取代的与氨基取代的折射率差不多. MOFs材料 中配体的多样性, 使其有望成为新一代的光学材料. 